\title{
The early development of self-injurious behaviour: Evaluating the role of social reinforcement.
}

\section{Oliver, C., Hall, S. \& Murphy, G.}

\section{Cerebra Centre for Neurodevelopmental Disorders, School of Psychology, University of Birmingham}

Please use this reference when citing this work:

Oliver, C., Hall, S. \& Murphy, G. (2005). The early development of self-injurious behaviour: Evaluating the role of social reinforcement. Journal of Intellectual Disability Research, 49, 591-599.

The Cerebra Centre for Neurodevelopmental Disorders,

School of Psychology, University of Birmingham, Edgbaston, Birmingham, B15 2TT

Website: www.cndd.Bham.ac.uk

E-mail: cndd-enquiries@contacts.bham.ac.uk 


\begin{abstract}
Background. The potential role of social reinforcement in the development of self-injury has not yet been subjected to empirical analysis. In this two year prospective study the pattern of social interactions related to the early presentation of self-injury were examined to identify a potential association with an increase in self-injury.

Method. The self-injurious behaviour and social contact with adults of sixteen children with intellectual disability with self-injury of recent onset were observed at three monthly intervals over two years.

Results. Increase in self-injury over a two year period was positively correlated with a distribution of social contact relative to episodes of self-injury that is consistent with a mutual social reinforcement paradigm. When this paradigm was operative self-injury was evoked under stable antecedent conditions over time but tended to evoke increasing levels of social interaction.
\end{abstract}

Conclusions. These results support the hypothesis that increases in the frequency of early self-injury in children with intellectual disabilities may be determined by social reinforcement with implications for early intervention and proactive identification of children at risk for increases in self-injury. 


\section{Introduction}

Self-injurious behaviour is shown by between $4 \%$ and $12 \%$ of children with intellectual disability with a marked increase in prevalence and severity between the ages of 5 and 25 (Oliver, Murphy and Corbett 1987). Self-injury may begin in children under the age of 5 (Berkson, Tupa and Sherman, 2001) and the limited data on chronicity suggest that selfinjury is intransigent in those whose behaviour is severe (Kebbon and Windahl, 1986; Murphy et al., 1993; Emerson, Kiernan and Alborz, 2001). In combination with models of the development of self-injury (Guess and Carr, 1991; Oliver, 1993, 1995; Kennedy, 2002) these findings suggest that an early intervention strategy warrants exploration. Such a strategy might be informed by study of the natural development of self-injury.

In the first study of children who had recently begun to show SIB, Murphy, Hall, Oliver and Kissi-Debra (1999) describe an increase in the frequency of SIB in four out of sixteen children over a two year period. Analysis of variables associated with SIB, such as severity of intellectual disability, physical and sensory impairment, (see McClintock, Hall and Oliver, 2003) showed no association with increase over two years. However, the sample was small and highly selected on the basis of some of these variables, thus limiting both power and variance. Observational data on the sixteen children recorded at three month intervals over two years were further analysed by Hall, Oliver and Murphy (2001). The analysis showed that for those children whose self-injury increased over two years, there was a higher probability of self-injury during periods when social contact low. This empirical observation warrants examination from a theoretical perspective that might explain the association between the raised probability of SIB when no social contact is occurring and the increase in SIB over the longer term. 
The majority of research into assessment and intervention for self-injurious behaviour draws on the principles of operant conditioning to identify the prevailing contingencies that maintain self-injury so that they might be beneficially modified. Using experimental and naturalistic methodology this research clearly indicates social reinforcement to be influential in the maintenance of self-injury at a given point in time (Lerman and Iwata, 1993; Hall and Oliver, 1992; Iwata, Pace, Dorsey et al., 1994). Social reinforcement of emerging and extant behaviours is also central to models of the development of self-injury over time. The stage model of Guess and Carr (1991) suggests that stereotyped behaviour, initially governed by levels of nonsocial sensory stimulation, evolves into self-injurious behaviour because of social reinforcement. Oliver $(1993,1995)$ describes a more fine grained model of the process of social reinforcement of SIB and proposes a mutual reinforcement paradigm similar to that described by Patterson (1982) for the development of conduct disorder. One explanation for the observation of Hall, Oliver and Murphy (2001), that long term increases in self-injury are associated with a higher probability of self-injury under conditions of low social contact, is that the low level of social contact evokes self-injury that is then socially reinforced in accordance with the mutual reinforcement paradigm described by Oliver (1995). The hypothesis that increases in self-injury over time are associated with the mutual reinforcement paradigm is the focus of this study.

Experimental investigation of all aspects of the mutual reinforcement paradigm is difficult because controlling each participant's behaviour to observe the effect on the other is wrought with methodological and ethical problems. To date support for the mutual reinforcement model comes from naturalistic observation studies (Lalli, Browder, Mace and Brown, 1993; Lerman and Iwata, 1993; Hall and Oliver, 1992). Interpreted using operant principles, the 
study of Hall and Oliver (1992) shows that a low level of social contact, an establishing operation of reinforcer deprivation, (see Michael; 1982) evokes self-injury which in turn evokes social contact (by virtue of the aversive property of self injury it is an establishing operation for social contact). As social contact is presented, self-injury ceases (as the establishing operation for self injury is abolished) and as self-injury ceases so social contact decreases (as the establishing operation for social contact is abolished). The cycle is then repeated. This pattern is consistent with maintenance of self-injury by positive reinforcement from social contact. In this cycle there is a predicted sequence to increases and decreases in social contact relative to the onset, presence and termination of episodes of self-injury. Identification of this sequence offers empirical support for the mutual reinforcement paradigm, although it does not conclusively establish that social contact is positively reinforcing or that the termination of self-injury is negatively reinforcing.

To test for the predicted sequence the normalise and pool method described by Hall and Oliver $(1997,2000)$ can be applied to the observational data on young children showing selfinjury described by Hall et al. (2001). Whilst this method does not examine the statistical significance of associations between observed behaviours, it does have the advantage of being able to deal with bursts of behaviour of differing lengths and focus on the overall distribution of social contact relative to a target behaviour whilst eliminating the constraints imposed by time based methods. It also has the advantage of enabling the simple presentation of a complex interaction in which a number of points warrant examination.

In this study it is predicted that for the children in the Hall, Oliver and Murphy (2000) study, an increase in SIB over a two year period will be positively associated with a distribution of social contact relative to SIB which is in accordance with a mutual reinforcement process 
assessed using normalise and pool analysis of the observational data. Specifically, for the positive social reinforcement hypothesis, SIB should occur when social contact is low, social contact should increase following the onset of SIB, the level of social contact should be higher after self-injury than before self-injury and social contact should decrease following the termination of self-injury. For demand escape motivated SIB, SIB should occur when demands are high, demands should decrease following the onset of SIB, the level of demands should be lower after self-injury than before self-injury.

\section{Method}

\section{Participants}

Detailed information on the sixteen children observed in the previous study are given in Murphy, Hall, Oliver and Kissi-Debra (1999) and Hall, Oliver and Murphy (2001). The age range was 2 years 6 months to 10 years 11 months (mean age 5.27 years, $\underline{S D}=1.94$ years). The mean developmental quotient (from the Vineland Adaptive Behavior Scales was 1.31 years $(\underline{\mathrm{SD}}=0.66$ years $)$. Seven of the children were male, eight had profound intellectual disability, seven were diagnosed as autistic and nine had poor or no ambulation. Six children had genetic disorders: Sturge-Weber (2), Cornelia de Lange (1), Seckel syndrome (1), Fragile X (1) and Trisomy 6Q (1). According to class teachers, the children had begun to show selfinjurious behaviours within the previous three month period. A variety of forms of SIB were shown by the children: head hitting (5), hand biting (5), head banging (3), hair pulling (2) body hitting (2) and scratching (1). Three children showed two forms of self-injury.

\section{Observational response definitions, recording, and interobserver agreement.}

Child responses recorded included head and body banging, head and body hitting, hand biting, scratching and rubbing, eye pressing and poking and hair pulling. Operational 
definitions for each of these responses can be found in Murphy, Hall, Oliver and Kissi-Debra (1999). Two teacher behaviours were also recorded: demands (defined as any verbal or physical direction by the teacher in order for the child to complete an action or task); attention (defined as any other physical or verbal contact made by the teacher to the child e.g., touching, response blocking, offering drinks or food, reprimanding and commenting). All responses were recorded on a laptop computer (Olivetti Quaderno, Model PT-XT-20) using software that allowed continuous documentation of the frequency and/or duration of each behaviour and their interrelations (see Repp, Harman, Felce, VanAcker, \& Karsh, 1989). Two observers (one standard observer) independently scored responses during $10 \%$ of observational data collected. Observer records were compared on a 10-s interval-by-interval basis, scoring agreements and disagreements on occurrence and non-occurrence for each recorded behaviour. The mean Kappa statistic was .68 (range, .59 to .74 ), suggesting that agreement between observers was acceptable.

\section{Procedure}

All children were observed in their school classroom on between four and eight occasions (mode of six) at approximately three month intervals (mean interval 3.67 months).

Classrooms normally contained the child's teacher, teaching assistants and between 4 and 10 other children. Each child in the study was observed for a 3 to 4 hour period at each observation point. Throughout each observation period, the observer followed the child unobtrusively and did not interact with the child. Teachers were reminded prior to each observation period to ignore the observer and interact normally with the child. Observations included a representative sample of activities: meals, group activities, individual work, and leisure time. 


\section{Descriptive analysis.}

In order to examine the overall distribution of social contact relative to bursts of SIB a method of analysis that determines the trend in the probability of social contact before, during and after SIB was employed (see Hall and Oliver, 1992, 1997, 2000). The method utilises all of the available data on social contact across the total observational time, not just time windows local to SIB, in order to evaluate trends over longer periods of time. To use all of the available data on the distribution of social contact relative to SIB the problem of the variability of the time that elapses between and within bursts of SIB must be addressed. This can be done by examining the distribution of social contact in the periods between and during bursts of SIB whilst controlling for difference in the length of the periods. When this is achieved the relative position of social contact to two bursts of SIB or within a burst of SIB can be identified regardless of the period of time that elapses between the two bursts of SIB or within the burst of SIB. So, if the distribution of social contact tends to peak prior to, midway between or following bursts of SIB this approach will identify this pattern regardless of the time that elapses between bursts of SIB. By using this approach it is therefore possible to examine the distribution of the probability of social contact relative to bursts of SIB and thus ascertain if the resultant profile is consistent with the mutual social reinforcement hypothesis.

To address the problem of the variability in time that elapses between bursts of SIB, the method "normalises" periods of time prior to, during and following occurrences of a nominal independent variable (in this case SIB) by dividing these periods into percentiles. The presence of a nominal dependent variable (in this case social contact) in each percentile in each period can then be ascertained. It is then possible to calculate the probability of social contact at each percentile and express these data as conditional probabilities of social contact 
given a burst of SIB. A summary diagram can then be constructed which encompasses all of the observational data collected and represents the probability that social contact precedes, occurs concurrently with and follows SIB.

The purpose of the diagrams is to consider the overall distribution of social contact relative to SIB and not the overall level (or base rate) of either behaviour as this is taken into account in the calculation of the probabilities. As it is the distribution of social contact relative to SIB that is of interest and not the level, the probability of social contacted is plotted on a y axis with minimum and maximum values that are equivalent to the minimum and maximum values across all percentile points. This allows the variability in trend in social contact to be identified regardless of the overall level or range of social contact. Thus, by using this diagram it is possible to compare the relationship between SIB and social contact across participants regardless of: the variability in the time that elapses between bursts of SIB, the burst length of SIB and the overall probability of social contact and SIB. Additionally, the diagrams document the relationship between the trend in social contact and the onset of SIB, the onset of SIB and the subsequent trend in social contact, the trend in social contact and the offset of SIB and the offset of SIB and the subsequent trend in social contact. Consequently, the mutual interplay between SIB and social contact is captured in a single plot to enable testing of the hypothesis.

\section{Results}

Change in SIB over two years for each participant was measured by the Beta coefficient derived from applying the linear growth model to the observed levels of SIB at each observation point. A positive value indicated an increase and a negative value a decrease (see 
Hall, Murphy and Oliver, 2001). The Beta coefficients varied from 1.10 to -.61. To evaluate the reciprocal social operant reinforcement process, summary normalise and pool plots of the probability of both teacher demands and attention prior to, during and following selfinjurious behaviour were produced for each participant.

Initial inspection of the plots for each child for demands and social contact considered separately revealed no difference between the profiles, thus suggesting that for these children at this time, demands have similar functional properties to attention ${ }^{1}$. Consequently, the teacher behaviours of demands and attention were combined to form a single behaviour of social contact and a single plot was constructed for each child using this behaviour. The resultant plots are shown in Figure 1. The plots are organised by the change in SIB over the two year period (greatest increase is top left, greatest decrease is bottom right) and the index of change (the Beta coefficient derived from applying the linear growth model) is shown for each child. Additionally, the plots indicate the minimum and maximum probability of social contact on the y axis relative to bursts of SIB.

\section{+++ Insert Figure 1 here +++}

The plots in figure 1 show that for nine children $(1,8,2,5,4,10,9,16)$ there is a decreasing level of social contact prior to the onset of SIB. For seven children $(1,8,2,4,10,3,9)$ the lowest probability of social contact when SIB is not occurring is immediately prior to the onset of SIB. For nine children $(1,8,2,7,10,12,6,3,9)$ there is an increase in social contact following the onset of SIB. For only two children $(1,2)$ there was a substantial increase in social contact following SIB in comparison to the period prior to SIB. In summary, two children $(1,2)$ evidenced a profile consistent with four features of a reciprocal positive social

\footnotetext{
${ }^{1}$ These plots are available from the corresponding author
} 
reinforcement process, three children $(8,10,9)$ evidenced three features, two children $(4,3)$ evidenced two features and the remaining nine children evidenced one or no features. Further analysis of the association between the number of features of a reciprocal positive social reinforcement process evidenced for a child and the Beta coefficient indicative of an increase over two years revealed a significant positive correlation $\left(\mathrm{r}_{\text {pearson }}(15)=.50, \mathrm{p}<.05\right)$. Thus an increasing number of indices of the mutual social reinforcement process is positively associated with an increase in SIB over a two year period in this group of children.

The two children whose normalise and pool plots evidenced all four features of a reciprocal social reinforcement process showed the highest and third highest increase in self-injury over the two year period. These two children were differentiated from others by the increase in the level of social contact between the periods prior to and following self-injurious behaviour. This indicates that a low level of social contact is associated with the onset of SIB and that SIB evokes social contact. This is in accordance with the mutual social reinforcement paradigm. Given this clear differentiation and its potential significance with regard to social positive reinforcement, further analysis of the change in the distribution of social contact prior to and following SIB over the two year period was conducted for these two children.

To examine change in the distribution of social contact relative to SIB throughout the two year period, normalise and pool plots for social contact around bursts of SIB for each child at each three month time point were derived. Two specific points in each plot were considered. These were the period immediately prior to the onset of SIB (corresponding to the antecedent for SIB) and the period immediately following the offset of SIB (corresponding to the consequence for SIB). If learning by both players in the mutual reinforcement paradigm is in effect, then over time it might be expected that SIB would be more likely to become 
associated with an antecedent (low social contact) and more evocative of the same consequence (the presence of social contact). Evidence for this would be increasing differentiation between levels of social contact before and after SIB over the two year time period. To examine this possibility two contingency indices were derived for each child at each time point. The first was an antecedent contingency index. This was calculated by dividing the difference between the level of probability of social contact at the onset of SIB (the mean of the last datum point immediately prior to the onset of SIB and the first datum point after the onset of SIB) and the lowest probability of social contact at any percentile during the observation period by the difference between the lowest and highest probability of social contact at any datum point during the observation period. Thus, the antecedent index indicated a level of social contact prior to SIB normalised for the unconditional probability of social contact which would vary within children over time and between children. Similarly, a consequence index was calculated by substituting the level of probability of social contact at the offset of SIB (the mean of the last datum point during SIB and the first datum point immediately following SIB) for the level of probability of social contact at the onset of SIB. These indices can vary from zero to one. A value of zero would indicate that the probability of social contact either immediately prior to or following SIB in comparison to all other times during the observations was at its lowest, conversely a value of one would indicate the probability of social contact is at its highest and these values can be compared between children and over time.

Figure 2 shows the antecedent and consequence contingency indices plotted for Child 1 and 2 at each observation and comparable data for two children (Child 11 and 8) whose normalise and pool plots did not show a change in the level of social contact prior to and following SIB but whose mean level of SIB was similar to Child 1 and 2. 
The plots of contingency indices shown in figure 2 show that for Child 1 and 2 there is an increase in relative probability of social contact between the periods immediately prior to and following SIB, thus confirming the stability of the plots in figure 1 over time. This differentiation is not evident for Child 11 and 8. Additionally, for Child 1 and 2 there is increasing differentiation between the probability of social contact prior to and following SIB over the two year period, although this increase is more systematic for Child 1 than Child 2. The pattern of this differentiation is the same for both Child 1 and 2; the relative level of probability of social contact for the antecedent period remains stable while the relative level of probability of social contact for consequent period increases. Thus for Child 1 and 2 there is evidence that whilst SIB is evoked under relatively stable antecedent conditions the behaviour is increasingly likely to evoke social contact over time when the unconditional probability of social contact is controlled for.

\section{Discussion}

To date the literature focussing on the early development of self-injurious behaviour has been restricted to theoretical models (Guess and Carr, 1991; Oliver, 1993, 1995; Kennedy, 2002), reports of prevalence and phenomenology (Berkson, Tupa, Sherman, 2001; Oliver, Murphy and Corbett, 1987) and empirical studies of associations with putative risk factors and the cooccurrence of environmental events (Murphy, Hall, Oliver and Kissi-Debra, 1999; Hall, Oliver and Murphy, 2001; McClintock, Hall and Oliver, 2003). In this study we have conducted an empirical examination of the association between an increase in the early development of SIB and observed characteristics of child-adult interactions that conform to a 
mutual social reinforcement paradigm (see Oliver, 1995). Two results are of significance for this paradigm. First, there is a positive correlation between an increase in early self-injurious behaviour and the number of features that indicate an adult-child interaction profile consistent with social positive reinforcement for SIB. Second, for two children who evidenced an increase in SIB and for whom the adult-child interaction profile was consistent with social positive reinforcement of SIB, there was an increase in the social contact that followed SIB over the two year period.

The first of these findings offers support to the models proposed by Guess and Carr (1991), Oliver (1993, 1995) and, more recently, Kennedy (2002). Central to each of these models is social reinforcement of SIB as explanatory of the transition from stereotyped behaviour, or proto-self-injury with a non-socially determined origin, to socially maintained SIB as described in contemporary experimental functional analytic studies (see Iwata et al., 1994). There are a number of implications of this finding. First, the emerging research into selfinjury in genetic syndromes should examine the applicability of the social reinforcement model to the behaviours purported to be behaviourally phenotypic (see Oliver, Murphy and Corbett, 1993; Hall, Oliver and Murphy, 2000). Although it should be acknowledged that more diverse determinants of SIB might be identified in some genetic syndromes. Second, early intervention strategies for self-injury must focus equally on contingency management strategies and the development of functionally equivalent behaviours such as functional communication (Carr and Durand, 1985a, 1985b). Functional communication, when effective, probably has the same evocative properties as socially determined SIB and thus may be subject to the mutual reinforcement process that cultivates maintenance and generalization (Horner and Day, 1991; Fisher, Piazza, Cataldo, Harrell, Jefferson et al., 1993). 
The second finding, that when the mutual social reinforcement process is operative the probability of contingent social contact increases over time, might be critical to understanding the increase in severity of SIB. It has been argued that SIB is reinforced because its aversive properties act as an establishing operation that evokes social contact from others (Oliver, 1995). If this is the case then the data presented in figure 2 suggest that the SIB is becoming increasingly aversive to others and thus is evoking higher levels of social contact. More aversive SIB may be more severe as it is more intense or damaging and thus a gradual shaping of more severe SIB might be embedded in the natural reinforcement processes (see Hall and Oliver, 1992; Oliver, 1993; Kennedy, 2002). The implication of this process is that when early SIB is functional then it has the capacity to naturally evolve into more severe forms unless an intervention is implemented.

Although these findings offer support for the role of a mutual social reinforcement process in the development of self-injury the results are not conclusive. The natural observation procedure and normalise and pool plots used to model the process cannot appraise whether social contact is reinforcing the SIB, nor can conventional lag sequential statistics be employed to evaluate the significance of the plots and some aspects of the analysis were not supported by quantitative analyses. The method and analysis were adopted for two reasons. First, to capture the mutual social reinforcement process the behaviour of neither participant can be controlled as the behaviour of each participant is both the independent and dependent variable simultaneously. Although experimental analogue methods might afford greater experimental control they require the behaviour of one participant in the dyad to be declared the dependent variable. Second, lag sequential analyses can typically only compare the unconditional probability of a behaviour to the conditional probability of a behaviour given 
another behaviour. This requires the imposition of arbitrary time windows, with a loss of information, and, in the case of Yule's Q, inappropriate assumptions about the requirement for both necessity and sufficiency of, for example, an antecedent for a behaviour. It is also notable that the mutual reinforcement process was not evident for many of the children and clearly other causes might be operative. This is exemplified by the number of children for whom only one or no features of the process were evident and the low proportion of the variance in increase in SIB over time accounted for in the correlational analysis. Future research might address these problems and seek to confirm the findings described here using a combination of natural observations with analogue conditions to validate the conclusions drawn from refined lag sequential analyses as they become available. 


\section{References}

Bakeman, R. \& Gottman, J.M. (1997). Observing Interaction: An Introduction to Sequential Analysis. Second Edition. Cambridge, Cambridge University Press.

Bakeman, R. \& Quera, V. (1995). Analyzing Interaction: Sequential Analysis with SDIS and GSEQ. Cambridge, Cambridge University Press.

Berkson, G., Tupa, M. \& Sherman, L. (2001). Early development of stereoyped and selfinjurious behaviors: I Incidence. American Journal on Mental Retardation, 106, 539-547.

Carr, E.G. and Durand, V. M. (1985a). The social-communicative basis of severe behavior problems in children. In S. Reiss and R. Bootzin (Eds.), Theoretical Issues in Behavior Therapy (pp. 219-254), Academic Press, New York.

Carr, E.G. and Durand, V. M. (1985b). Reducing behavior problems through functional communication training. Journal of Applied Behavior Analysis, 18, 111-126.

Durand, V.M. \& Carr, E.G. (1991). Functional communication training to reduce challenging behavior: Maintenance and application in new settings. Journal of Applied Behavior Analysis, 24, 251-264.

Emerson, E., Kiernan, C., Alborz, A. (2001). Predicting the persistence of severe selfinjurious behavior. Research in Developmental Disabilities, 22, 67-75.

Fisher, W. , Piazza, C., Cataldo, M., Harrell, R., Jefferson, G. \& Conner, R. (1993). Functional communication training with and without extinction and punishment. Journal of Applied Behavior Analysis, 26, 23-36.

Guess, D. \& Carr, E.G. (1991). Emergence and maintenance of stereotypy and self-injury. American Journal on Mental Retardation, 96, 299-319.

Hall, S. \& Oliver C. (1992). Differential effects of severe self-injurious behaviour on the behaviour of others. Behavioral Psychotherapy, 20, 355-365. 
Hall, S., Oliver, C. \& Murphy, G. (2001). Early development of self-injurious behavior: An empirical study. American Journal on Mental Retardation, 106, 189-199.

Hall, S \& Oliver, C. (1997). A graphical method to aid the sequential analysis of observational data. Behavior Research Methods, Instruments \& Computers, 29, 563-573.

Hall, S. \& Oliver, C. (2000). An alternative approach to the sequential analysis of behavioral interactions. In: T. Thompson, D. Felce and F. Symons (Eds.), Behavioral observation: Technology and applications in developmental disabilities. (pp. 335-348). Baltimore: Paul H. Brookes.

Horner, R. H. \& Day, H. M. (1991). The effects of response efficiency on functionally equivalent competing behaviors. Journal of Applied Behavior Analysis, 24, 719-732.

Iwata, B.A., Pace, G.M., Dorsey, M.F., Zarcone, J.R., Vollmer, T.R., Smith, R.G., Rodgers, T.A., Lerman, D.C., Shore, B.A., Mazaleski, J.L., Goh, H., Cowdery, G.E., Kalsher, M.J., McCosh, K.C. \& Willis, K.D. (1994). The functions of self-injurious behavior: An experimental epidemiological analysis. Journal of Applied Behavior Analysis, 27, 215-240.

Kebbon, L. \& Windahl, S.I. (1986). Self-injurious behavior: Results of a nationwide survey among mentally retarded persons in Sweden. In: J.M. Berg \& J.M. Dejong (Eds.), Science and Service in Mental Retardation (pp. 142-148). London, Methuen.

Kennedy, C.H. (2002). Evolution of stereotypy into self-injury. In: S.R. Schroeder, M.L. Oster-Granite \& T. Thompson, Self-injurious behavior: Gene-brain-behavior relationships (pp.133-144). American Psychological Association: Washington DC.

Lalli, J.S., Browder, D.M., Mace, F.C. \& Brown, D.K. (1993). Teacher use of descriptive analysis data to implement interventions to decrease students' problem behaviors. Journal of Applied Behavior Analysis, 26, 227-238.

Lerman, D.C. \& Iwata, B.A. (1993). Descriptive and experimental analyses of variables maintaining self-injurious behavior. Journal of Applied Behavior Analysis, 26, 293-319. 
McClintock, K., Hall, S. \& Oliver C. (in press). Risk markers for challenging behaviour in people with intellectual disabilities: A meta-analytic approach. Journal of Intellectual Disability Research.

Michael, J. (1982). Distinguishing between discriminative and motivational functions of stimuli. Journal of the Experimental Analysis of Behavior, 37, 149-155.

Murphy, G., Hall, S., Oliver, C. \& Kissi-Debra, R. (1999). Identification of early selfinjurious behaviour in young children with intellectual disability. Journal of Intellectual Disability Research, 43, 149-163.

Oliver, C. (1993). Self-injurious behavior: from response to strategy. In C. Kiernan (Ed.), $\underline{\text { Research to Practice? Implications of Research on the Challenging Behavior of People with }}$ Learning Disability (pp. 136-188). Kidderminster: BILD Publications.

Oliver, C. (1995). Self-injurious behavior in children with learning disabilities: Recent advances in assessment and intervention. Journal of Child Psychology and Psychiatry, 30, 909-927.

Oliver, C., Murphy, G.H. \& Corbett, J.A. (1987). Self-injurious behavior in people with mental handicap: A total population study. Journal of Mental Deficiency Research, 31, 147162.

Patterson, G. R. (1982). Coercive Family Process. Eugene OR: Castalia.

Repp, A.C., Harman, M.L., Felce, D. Van Acker, R. \& Karsh, K.G. (1989). Conducting behavioral assessments on computer collected data. Behavioral Assessment, 11, 249-268.

Willett, J.B. (1988). Questions and answers in the measurement of change. In E.Z. Rothkopf (Ed.), Review of Research in Education (Vol. 15, pp. 345-422). Washington, DC: American Educational Research Association. 


\section{Acknowledgements}

We are very grateful to the children, their parents and teachers who helped in this study. This research was funded by the Mental Health Foundation, UK. 


\section{Legend for figures}

Figure 1. Normalise and pool plots showing the probability of social contact before, during and after bursts of self-injurious behaviour for each child ( $\mathrm{C} 1$ to $\mathrm{C} 16)$. Observational data have been pooled across all observations. The lowest and highest conditional probability values for percentile points are shown on the y axis and datum points are plotted between these values to show the distribution of social contact relative to self-injurious behaviour and the position of the lowest and highest conditional probability values of social contact relative to the onset and termination of bursts of self-injurious behaviour. The beta coefficient is also shown for each child indicating an increase (positive values) and decrease (negative values) in self injury (see text).

Figure 2. Values of contingency indices for two children whose normalise and pool plots evidenced a profile consistent with a mutual social reinforcement process (Child 1 and 2) and two children with comparable levels of self-injury demonstrating increasing differentiation between antecedent and consequent levels of social contact over time when social reinforcement is operative (see text). 

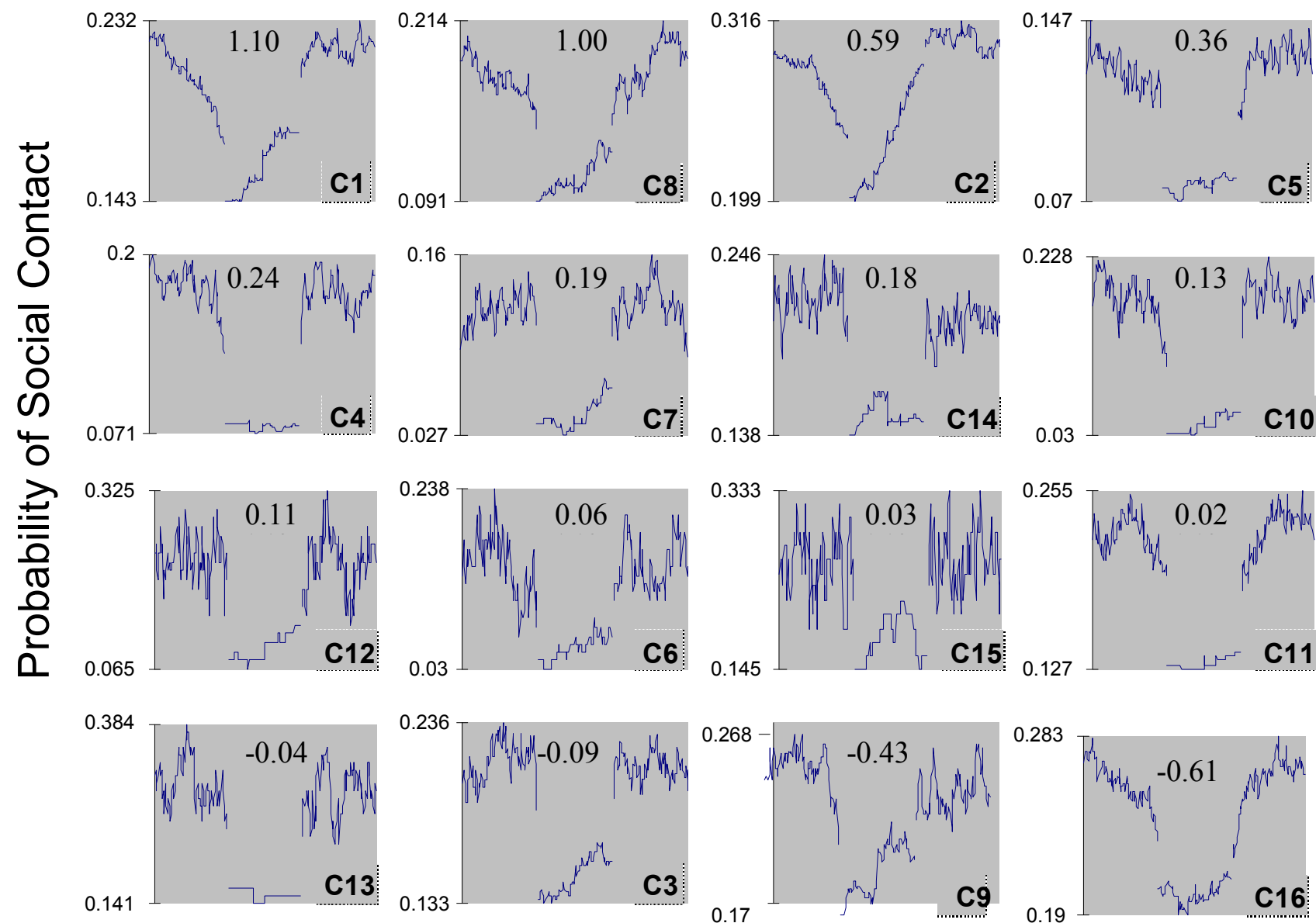

\section{Percentiles of Time}

Before, During, and After SIB 

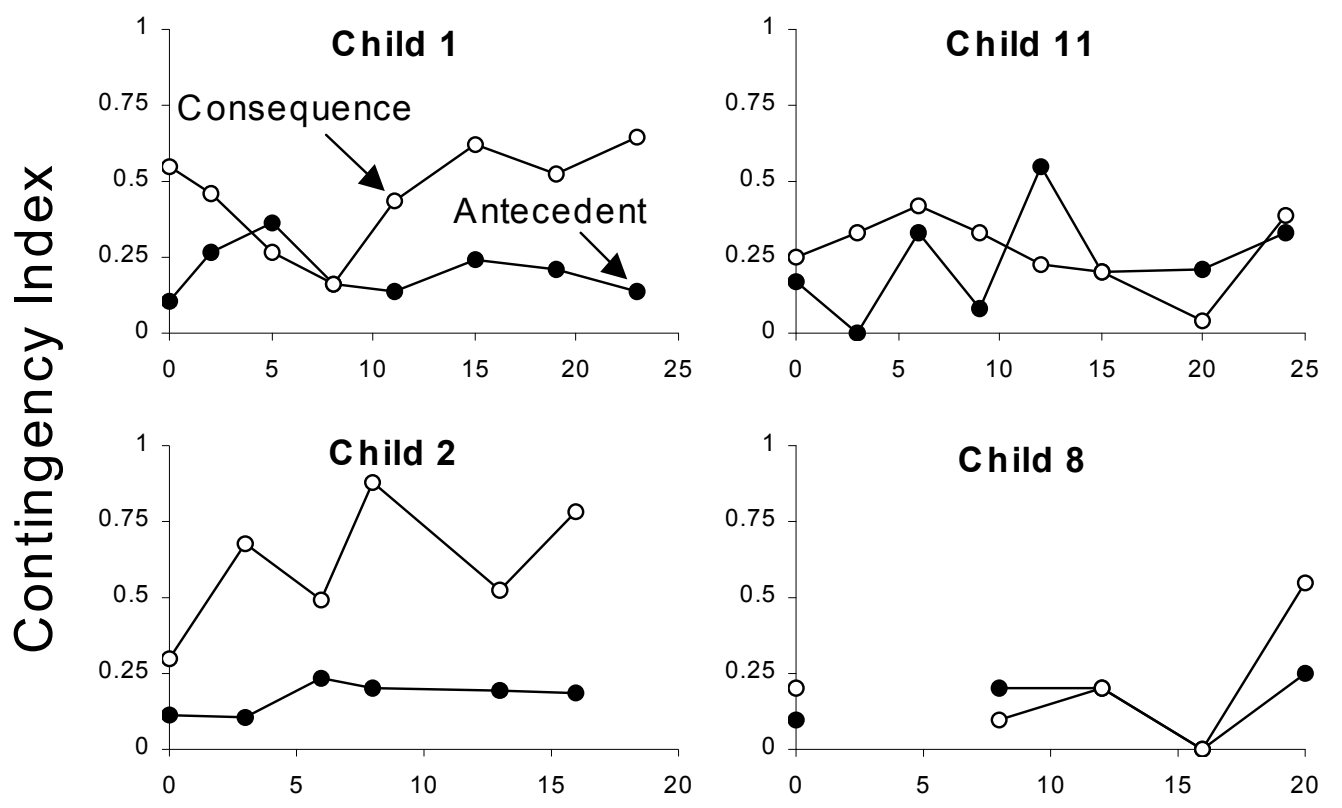

Time (months) 